\title{
A mathematical morphology approach to the identification of drought events in space and time
}

\author{
H. Vernieuwe ${ }^{\mathrm{a}}$, B. De Baets ${ }^{\mathrm{a}}$, N.E.C. Verhoest ${ }^{\mathrm{b}, *}$ \\ ${ }^{a}$ KERMIT, Department of Data Analysis and Mathematical Modelling, Ghent \\ University, Coupure links 653, 9000 Ghent, Belgium \\ ${ }^{b}$ Laboratory of Hydrology and Water Management, Ghent University, Coupure links 653, \\ 9000 Ghent, Belgium
}

\begin{abstract}
Drought events occur worldwide and possibly incur severe consequences. Trying to understand and characterizing drought events is of primordial importance in order to improve the preparedness for coping with future events. In this paper, drought events are characterized by exploiting their spatiotemporal nature. Operators borrowed from mathematical morphology are applied to represent drought events as connected components in space and time. Characteristics reflecting the affected area, duration, and intensity are extracted from the proposed representation of a drought event. As an illustration, drought events are identified on the basis of a 35-year data set of daily soil moisture values covering Australia.
\end{abstract}

Keywords: mathematical morphology, drought identification, space and time, connected component

\section{1. Introduction}

Drought events are caused by a lack of precipitation over a large area and a long period of time. On-site, it is relatively easy to tell whether or not one is experiencing a drought event. Yet, properly defining a drought event is not an easy task. A drought event can be regarded as a creeping hazard with no clear start and ending, it furthermore moves around and changes in space and time. Drought events occur worldwide and sometimes have severe

\footnotetext{
${ }^{*}$ Corresponding Author

Email addresses: hilde.vernieuwe@ugent.be (H. Vernieuwe), bernard.debaets@ugent.be (B. De Baets), niko.verhoest@ugent.be (N.E.C. Verhoest)
}

Non-peer reviewed preprint submitted to EarthArXiv and Elsevier 
socio-economic consequences. At the worst, they cause catastrophes such as famines and may be the immediate reason of severe conflicts or even wars. In order to improve the preparedness for future drought event occurrences by e.g. adjusting or drawing up water management plans, it is essential to first try to understand and characterize these phenomena.

In this paper, an attempt is undertaken to characterize drought events by exploiting their spatio-temporal nature. Operators borrowed from mathematical morphology (Serra, 1986) are first applied such that drought events can be represented as connected components in space and time. Next, of the resulting events, characteristics describing the affected area, duration and intensity are extracted. This drought event representation and characterization can serve as a support for water managers and users in the field. Future research will then focus on the comparison of an ongoing drought event with historical drought events on the basis of the delineated characteristics.

In scientific literature, much effort has already been made to try to quantitatively characterize drought events. However, capturing all aspects of a drought event is still a difficult task, as it concerns a moving and changing phenomenon. Tallaksen and Van Lanen (2004) pointed out that a drought event is a spatio-temporal phenomenon of which the space- and time-components are to be considered. Yet, only recently an increasing number of studies have reported on the characterization of drought events as a spatio-temporal connected component. Andreadis et al. (2005) were among the first to introduce a spatial identification procedure and took into account the fact that multiple drought events at one time step can merge into a larger drought event at a subsequent time step. Similarly, they consider that one single drought event can break up into multiple smaller drought events. Sheffield et al. (2009) also employed this spatial identification procedure for drought area identification, whereas Lloyd-Hughes (2011) further elaborated on this procedure and extended it from the spatial domain to the space-time domain to extract coherent space-time structures. Herrera-Estrada et al. (2017) also employed the method of Andreadis et al. (2005) to try to track the movement of drought events across regions. In a parallel path of trying to understand the impact of climate extremes on terrestrial ecosystems and corresponding land-atmosphere fluxes, Zscheischler et al. (2013) identified large spatio-temporal contiguous extreme events by identifying connected components through the use of a flood-fill algorithm.

Generally, whenever drought events are characterized, one relies upon drought indices such as the popular standardised precipitation index (McKee et al., 1993) or the Palmer drought severity index (Palmer, 1965) in order to be able to evaluate drought characteristics of interest (Mishra and Singh, 
2010). Next, relationships between drought characteristics such as severityintensity-duration or severity-area-duration are generally established (see, among others, the work of Sridhar et al. (2008); Mishra et al. (2009)), some by means of copulas (e.g. Wong et al. (2010)), such that the dependence between characteristics can be summarized, thus facilitating a frequency analysis of the events by means of return periods (see e.g. Halwatura et al. (2015); Reddy and Ganguli (2011); Serinaldi et al. (2009)). Nevertheless, one has to bear in mind that all drought indices have their own advantages and shortcomings (Sheffield et al., 2004; Sheffield and Wood, 2011). Applications of these indices may hence also suffer from these inadequacies (Sheffield et al., 2004). Furthermore, Sheffield and Wood (2007, 2011) suggest to use percentile values of a drought variable in order to overcome inconsistencies between severity classes assigned by different drought indices and to provide a sound probabilistic grounding for a drought index. As percentile values represent probabilities of occurence, using percentile values also allows for a straightforward comparison between values at different locations (Sheffield and Wood, 2007, 2011).

Sheffield et al. (2004) and Sheffield and Wood (2007) state that soil moisture is a useful drought index. It is a key variable in the hydrological cycle as it controls the majority of processes in the hydrological cycle such as evaporation, runoff, infiltration and drainage. It also reflects the impact of meteorological variables such as temperature and radiation. Furthermore, soil moisture values in the top layer of the soil are related to short-term precipitation, whereas soil moisture values in the root zone indicate the amount of water available for plant growth, and soil moisture values in the deeper soil layers represent how much water is available for recharge to aquifers and rivers (Sheffield et al., 2004).

Hence, in the present study, drought characteristics are determined on the basis of percentile values of soil moisture. Furthermore, a long time series of spatial soil moisture data is required, such that the spatial and temporal components of a drought event can be captured. A time series of ca. 35 years of daily values of GLEAM-estimated (Miralles et al., 2011; Martens et al., 2017) soil moisture data at a resolution of $0.25^{\circ}$ meets these requirements and is employed in this study. As Australia is regularly affected by drought events, data covering Australia were selected from this global data set. It was decided to focus on daily values in contrast to the generally-used monthly values, as in this way drought events that last less than one month and are terminated by a single storm (Byun and Wilhite, 1999; Sheffield et al., 2004) can still be detected. Furthermore, drought events are identified in space and time using operators from mathematical 
morphology (Serra, 1986). Further, characteristics reflecting the spatial and temporal components and the severity level of the drought events are determined. In order to make a small comparison to the general approach of using monthly data, the method employing operators from mathematical morphology to identify drought events in space and time will also be applied to weekly data, such that drought events with a minimal duration of $c a$. one month will be retained.

Section 2 first elaborates on the data and the study region chosen for this research. Section 3 then explains the data pre-processing, the selection of the threshold used as a basis for identifying drought events and illustrates the basics of mathematical morphology and its application in the identification of drought events. Section 4 further elaborates on the determined characteristics of the resulting drought events. Section 5 then formulates the conclusions that are drawn from the results.

\section{Data and study region}

In order to be able to characterize an ongoing drought event, one has to be able to compare it to historical drought events. To that end, a long time series of historical data is required. Ideally, such time series should be available at large scales in order to also capture the spatial characteristics of the events. With the emergence of satellite remote sensing data in the late seventies, obtaining information at a high temporal and spatial resolution has become easier. The Global Land Evaporation Amsterdam Model (GLEAM) (Miralles et al., 2011) maximally benefits from the use of satellite-derived observations to estimate terrestrial evaporation and soil moisture. Its resulting data sets of evaporation and soil moisture have already been used in modelling studies or in evaluations w.r.t. other data sets (see e.g. Tobin and Bennett (2017); Roy et al. (2017); Majozi et al. (2017); Lopez et al. (2017); McCabe et al. (2016); Liu et al. (2016); Lorenz et al. (2014); Trambauer et al. (2014)). The data set employed in this paper (the GLEAM v3.0a data set (Martens et al., 2017)) spans a period of 35 years (from 1/1/1980 till 31/12/2014) of global daily root-zone soil moisture values at a $0.25^{\circ}$ resolution. The data has been estimated on the basis of satellite-observed soil moisture, vegetation optical depth and snow water equivalents, reanalysis air temperature and radiation and a multi-source precipitation product (Martens et al., 2017) and shows a slightly higher quality compared to other GLEAM data sets when evaluated against in situ measured soil moisture data. The depth of the root zone employed in GLEAM is a function of the land-cover type and can consist of up to three layers 
(0-10 cm, 10-100 cm and 100-250 cm). Three (resp. two) layers are taken into account for the fraction of tall (resp. small) vegetation, whereas only the first layer is taken into account for the bare soil fraction.

As Australia is vulnerable to the effects of climate change, in particular to the expected drying trend for the next 50-100 years (McCarthy et al., 2001), daily data covering Australia were selected from this GLEAM data set. At present, substantial agricultural areas are affected by periodic drought events and Australia contains large areas of arid and semi-arid land. Unlike in many other drought characterization studies, it was decided not to convert the daily values to monthly values as these daily values will allow for the detection of drought periods lasting less than one month. As pointed out by Byun and Wilhite (1999), an affected drought region can return to normal conditions with only one day's rainfall.

\section{Data pre-processing and drought identification}

As one of the drought characteristics that will be determined throughout this paper is linked to the spatial component of the event, the soil moisture values were first reprojected to the Lambert Azimuthal Equal Area coordinate system such that areas can be accurately calculated. These reprojected data, with a resolution of $27.442 \mathrm{~km} \times 29.079 \mathrm{~km}$, are then further used as the basis for the drought characterization in the remainder of the paper.

\subsection{Selection of the drought threshold}

As pointed out by Sheffield and Wood (2011), characterizing a drought event is a challenging task because it varies in many dimensions: its spatial components describe the area it covers, its temporal component reflects the time it persists and its intensity changes both in space and time. Ideally, the characterization of a drought event should reflect all of these components and should furthermore be transferable across regions and through time. Therefore, the suggestion of Sheffield and Wood (2007) and Sheffield and Wood (2011) to use a percentile level as threshold for defining a drought event is followed in this paper. A value below the threshold then indicates that drought conditions are met. A value of $10 \%$ was chosen, which then reflects that drought conditions are observed $10 \%$ of the time. This value can also be regarded as the value that separates moderate from severe and more extreme drought events (Andreadis et al., 2005). Furthermore, to take into account that a drought event is not restricted to a single location and to allow that the soil moisture values of the neighbouring locations also take 
part in determining the soil moisture value of the threshold, a neighbourhood was identified around the location under consideration. In this research study, three different sizes of neighbourhoods $\mathrm{N}$, i.e. a $3 \times 3-, 5 \times 5$-, and $7 \times 7$-neighbourhood, were used. In this way, a smoother transition between the soil moisture values of the thresholds for neighbouring pixels is established. In order to set the soil moisture value corresponding to the percentile threshold of $10 \%$ for the pixel at hand, the empirical cumulative distribution function of all soil moisture values observed within this neighbourhood was established. In this way, determining a threshold corresponding to the 10th percentile indicates that the soil moisture value at the location should drop below the soil moisture value corresponding to the 10th percentile of the entire neighbourhood before it is regarded as dry. This idea can easily be extended when one aims at taking into account larger neighbourhoods such as regions with the same land cover.

\subsection{Applicaton of mathematical morphology}

After selecting only those pixels with a value below the 10th percentile value a time series of binary maps that indicate which locations possibly belong to a drought event are obtained (see Figure 1 for an example of such a map for a $3 \times 3$-neighbourhood). It is clear that applying the threshold results in a scattered pattern. Single pixels are denoted as dry, while larger dry regions contain pixels that are not denoted to be dry. This also occurs in the time-dimension. Pixels can be denoted as dry for one time step, whereas for some following time steps, they are denoted as not dry, followed by being denoted as dry in the time steps thereafter. Hence, a processing procedure is required in order to smooth away these irregularities. A method that is well suited for this purpose is mathematical morphology (Serra, 1986), whose operations aim at simplifying images by retaining the essential shape characteristics and removing irrelevancies (Haralick et al., 1987). Applications and extensions of mathematical morphology w.r.t. image filtering, image segmentation, etc. have already been reported in the processing of remote sensing data (Soille and Pesaresi, 2002) and medical image analysis (Dufour et al., 2013).

By using the basic operators from mathematical morphology, i.e. erosion and dilation, the salt-and-pepper noise, i.e. the holes within the larger drydenoted regions and the smaller dry-denoted regions can be filled or removed. To apply these operators, a structuring element should first be determined, the size of which influences the size of the dry-denoted regions that will be removed and the holes that will be filled. As a drought event has a spatiotemporal character, it is chosen to employ a structuring element that has 


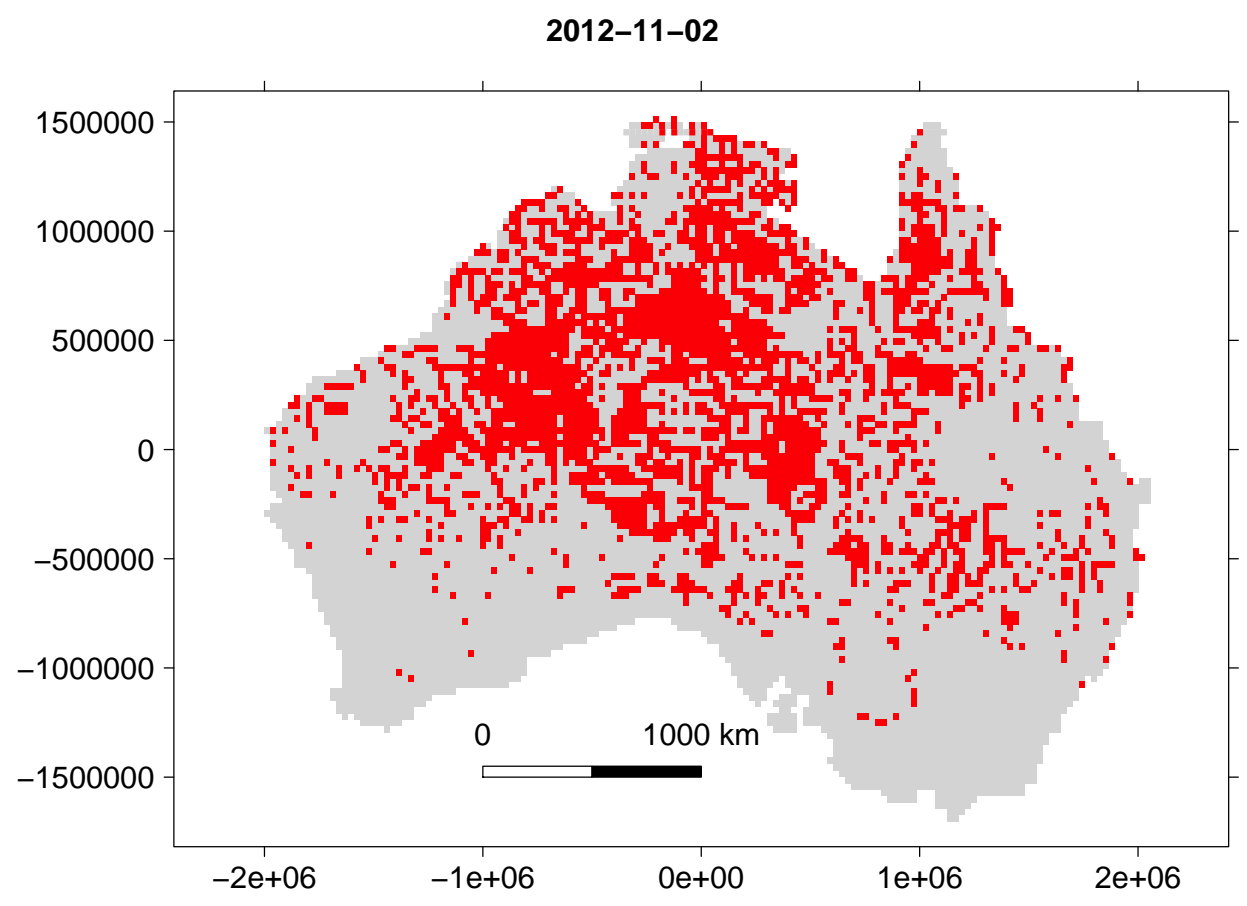

Figure 1: Possible drought locations (red) after thresholding a percentile map

space-time dimensions. To this end, the thresholded maps of the time series are placed one after the other, and a three-dimensional structuring element can hence be applied to this series. Different sizes of structuring elements were used of which the smallest a $3 \times 3 \times 3$ - and the largest a $7 \times 7 \times 7$-box. The first two dimensions indicate the spatial size of the structuring element, the last one indicates the number of time steps that is taken into account.

For reasons of simplicity, the erosion and dilation operators are first explained in two dimensions. Consider the space $E$ of all pixels, of which the set $A$ of dry-denoted pixels at a particular time step is a subset. With each point $x$ of the space $E$, a two-dimensional structuring element $B(x)$, e.g. a square, is associated. Figure $2(\mathrm{a})$ shows the space $E$, the set $A$ of drydenoted pixels indicated in grey and a $3 \times 3$ structuring element (red). The 
erosion $\epsilon$ of $A$ and dilation $\delta$ are then:

$$
\begin{aligned}
\epsilon(A) & =\{x \in E \mid B(x) \subseteq A\}, \\
\delta(A) & =\{x \in E \mid B(x) \cap A \neq \emptyset\} .
\end{aligned}
$$

The application of the above operators on $A$ is performed as follows. The structuring element is positioned with its center at each pixel $x$ of $E$, i.e. $B(x)$. Regarding the erosion operator, a grey pixel $x$ will remain grey if the pixels covered by the structuring element, positioned at $x$, are a subset of $A$. Regarding the dilation operator, a white pixel $x$ becomes grey if the intersection of the pixels covered by the structuring element positioned at $x$ with $A$ is not empty. Figures 2 and 3 illustrate these two morphological operators. An original map, for which the dry-denoted pixels (the set $A$ ) are indicated in grey, is given in Figure 2 (a) together with the $3 \times 3$ structuring element (red). In Figure 2(b), the structuring element is positioned at the pixel corresponding to its central pixel, indicated in light grey. As by this positioning, the pixels covered by the structuring element do not make up a subset of dry-denoted pixels of the original map, this pixel remains white, i.e. not dry, after the erosion process. Similarly, the pixel corresponding to the structuring element's central pixel in Figure 2(c) will remain grey, i.e. dry, after the erosion process, as for this pixel, the set of pixels covered by the structuring element is a subset of the original set. Figure 2(d) shows the final result after positioning the structuring element at all pixels of the space $E$. Analogously, Figure 3 illustrates the application of the dilation operator.

The compositions $\gamma=\delta \circ \epsilon$ and $\phi=\epsilon \circ \delta$ are called the morphological opening and closing, respectively. By first applying a morphological opening followed by a morphological closing, an open-close filter is obtained and the salt-and-pepper noise can be removed in the following sequential way:

$$
\begin{aligned}
\epsilon(A) & =\{x \in E \mid B(x) \subseteq A\} \\
\gamma(A) & =(\delta \circ \epsilon)(A)=\{x \in E \mid B(x) \cap \epsilon(A) \neq \emptyset\} \\
(\delta \circ \gamma)(A) & =\{x \in E \mid B(x) \cap \gamma(A) \neq \emptyset\} \\
(\phi \circ \gamma)(A) & =(\epsilon \circ \delta \circ \gamma)(A)=\{x \in E \mid B(x) \subseteq(\delta \circ \gamma)(A)\} .
\end{aligned}
$$

For applying the above-described operators in the three dimensions of the time series of thresholded images, the space $E$ now consists of all pixels at all time steps in this time series, and $A$ is the time series of dry-denoted pixels. The three-dimensional structuring element, e.g. a $3 \times 3 \times 3$-box, is then positioned at each pixel $x$ in $E$, and the operators can be applied as 


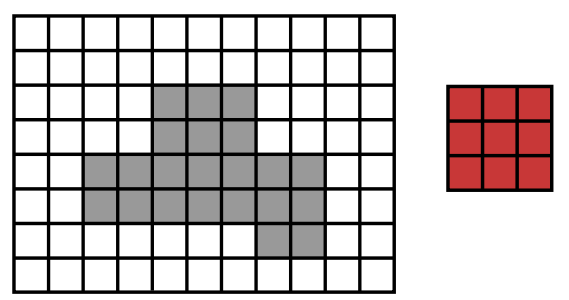

(a)

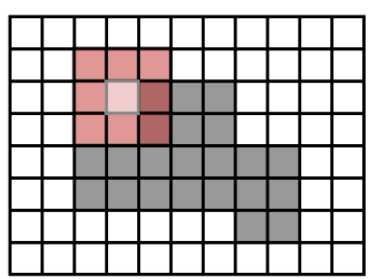

(b)

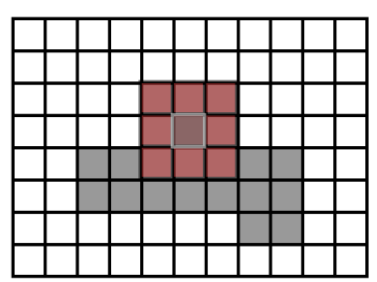

(c)

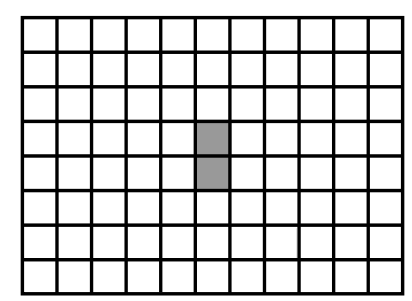

$(d)$

Figure 2: Map and structuring element (a), two steps (b) and (c) picked out of the erosion process and result (d) of the erosion process. 


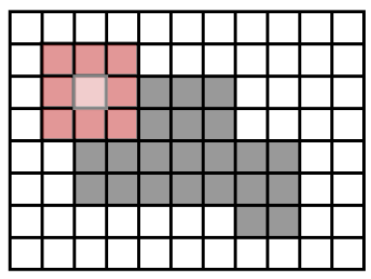

(a)

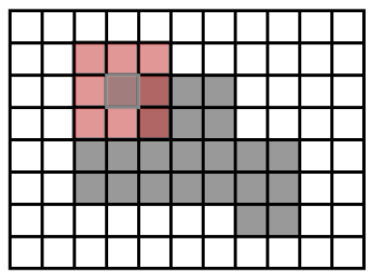

(b)

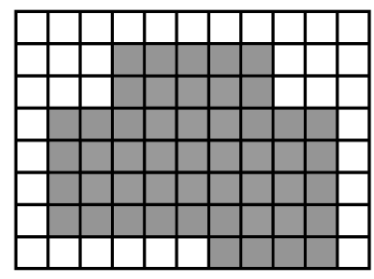

(c)

Figure 3: Two steps (a) and (b) picked out of the dilation process on the image in Fig 2(a) and result (c) of the dilation process. 
described before. By first applying the erosion operator (see Eq. (3)), drydenoted regions that are strictly smaller than the structuring element are removed. Larger dry-denoted regions are diminished and existing holes will be, initially, enlarged. By applying the dilation operator (see Eq. (4)) in the second step, the diminished regions are enlarged and the enlarged holes are diminished. The application of the dilation and erosion operator in the third and fourth step, respectively (see Eq. (5) and Eq. (6)), aims at removing holes that are strictly smaller than the structuring element.

The resulting dry-denoted regions that are connected in space and time are then regarded as separate drought events. An operational definition of a drought event is thus obtained:

\section{A single drought event is defined as a connected component in space and time after application of the morphological operators.}

Figure 4 illustrates the resulting drought events obtained at November the 2nd, 2012, after applying an open-close filter to the thresholded time series with a $3 \times 3 \times 3$-box. Different colors are used to illustrate the different drought events. This figure clearly shows that at the given time step, the green-coloured drought event is not spatially contiguous. However, as in former or later time steps, the currently isolated green parts merge, these parts belong to the same drought event. For the GLEAM data set spanning 35 years, 1859 drought events were identified in this way. The smallest and shortest drought event of these identified events corresponds to the size of the chosen structuring element. It should be noted however, that by enlarging the size of the structuring element to e.g. a $5 \times 5 \times 5$-box, fewer drought events will be identified of which the smallest drought event will hence correspond to the size of this applied structuring element. Furthermore, as the dilation operator is part of the procedure to identify drought events, it is inevitable that pixels that are originally denoted as not dry, i.e. their value is higher than the threshold, will become part of the identified drought event.

It is furthermore to be noted that by applying a larger structuring element, e.g. a $7 \times 7 \times 7$-box, larger parts of a possible drought event will be eliminated when the structuring element does not fit between the holes, i.e. the salt noise, in the event. This means that in the application of the erosion operator (Eq. (3)), the structuring element does not entirely overlap the dry-denoted pixels resulting in the removal of these pixels. For instance, after application of an open-close filter with a $7 \times 7 \times 7$-box on the thresholded time series for the date corresponding to Figure 1, no drought event 


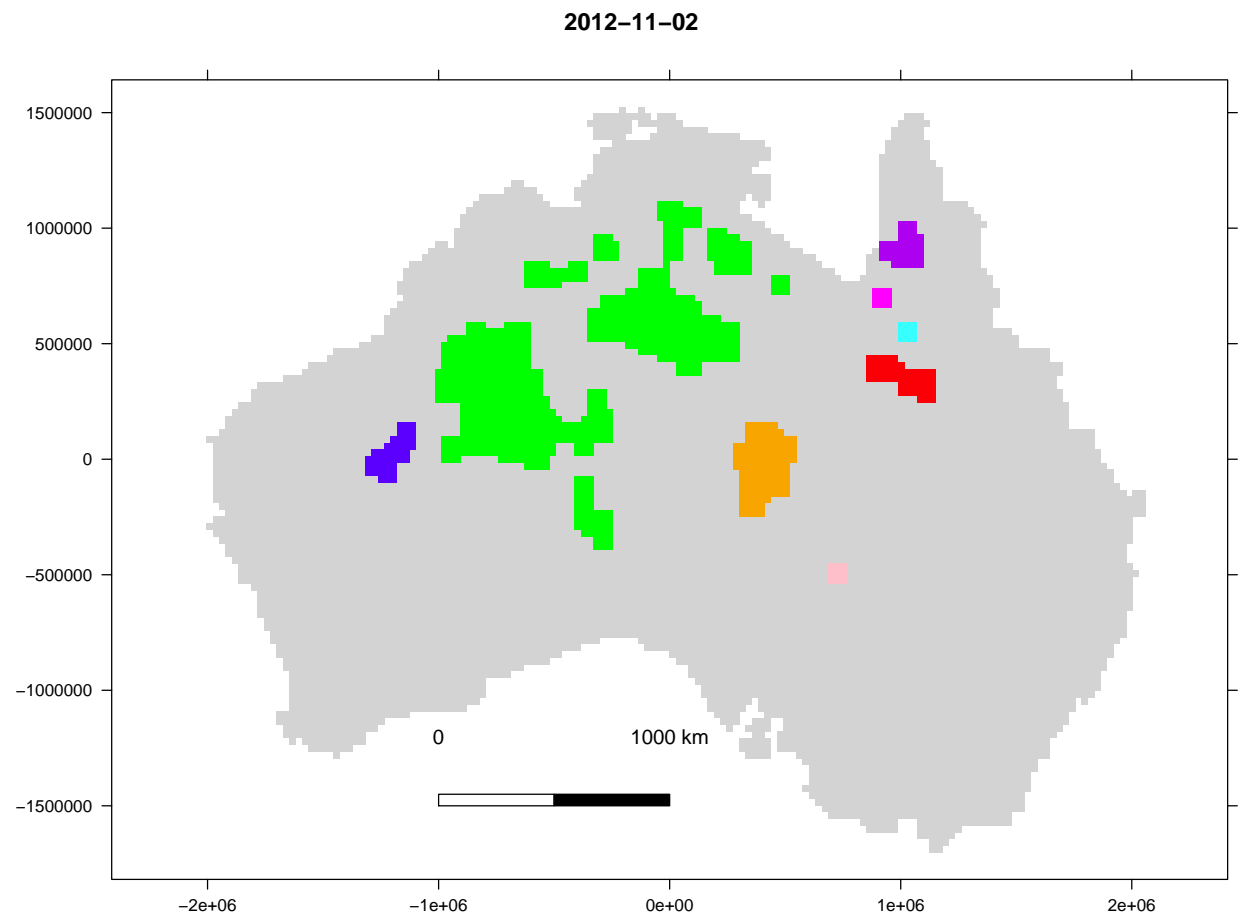

Figure 4: Drought events identified after application of an open-close filter to the thresholded time series. Different colours indicate different drought events. From the drought events identified, only one day is shown as illustration. 
was identified. Such a result is not desired as Figure 1 clearly shows that a quite large contiguous area, apart from some isolated pixels, is dry. This side-effect can be alleviated by gradually eliminating the noise components by using an open-close filter sequentially, starting from a small structuring element in the first iteration and by enlarging the structuring element for subsequent iterations. Such filter is called an alternating sequential filter (ASF) (Serra and Vincent, 1992):

$$
\mathrm{ASF}_{i}=\left(\phi_{i} \circ \gamma_{i}\right) \circ\left(\phi_{i-1} \circ \gamma_{i-1}\right) \circ \ldots \circ\left(\phi_{1} \circ \gamma_{1}\right),
$$

in which $i$ represents the $i$-th iteration, $\gamma_{1}$ and $\phi_{1}$ represent the opening, respectively closing operator with the smallest structuring element. $\mathrm{ASF}_{1}$ hence corresponds to the open-close filter using a $3 \times 3 \times 3$-box. ASF-filters were applied in order to compare the results of directly applying a larger structuring element to the results of gradually enlarging the structuring element, i.e. by applying $\mathrm{ASF}_{2}$ and $\mathrm{ASF}_{3}$ respectively with a $3 \times 3 \times 3$ and a $5 \times 5 \times 5$ - and a $3 \times 3 \times 3$-, a $5 \times 5 \times 5$ - and a $7 \times 7 \times 7$-box. Figure 5 illustrates the obtained result after applying $\mathrm{ASF}_{2}$ and $\mathrm{ASF}_{3}$ on the time series resulting from a $3 \times 3$-neighbourhood. It can be seen that the larger drought event (green-coloured in Figure 4) also appears in the result after applying $\mathrm{ASF}_{3}$. This favours the application of an ASF-filter instead of directly applying an open-close filter with a larger structuring element. The splitted drought event, purple-coloured in the top panel of Figure 5, is identified by $\mathrm{ASF}_{2}$, however, its upper part is too small to be identified by $\mathrm{ASF}_{3}$. Furthermore, it is noted that the larger drought event (green-coloured in Figure 4) has been split in smaller events (green- and purple-coloured in Figure 5) after application of an ASF. The next sections will further elaborate on the influence of the size of the structuring elements or the filter used (open-close vs. ASF).

\section{Drought characteristics}

For each drought event identified by the above-described method, characteristics reflecting its spatial and temporal components and its intensity level are determined. With respect to the spatial component, one needs a characteristic that summarizes the extent reached by the event. The maximal area covered by the drought event could characterize the affected area. However, it might be more informative to aggregate the $\tau$ largest daily areal extents. In order to be in line with the size of the structuring elements 
2012-11-02

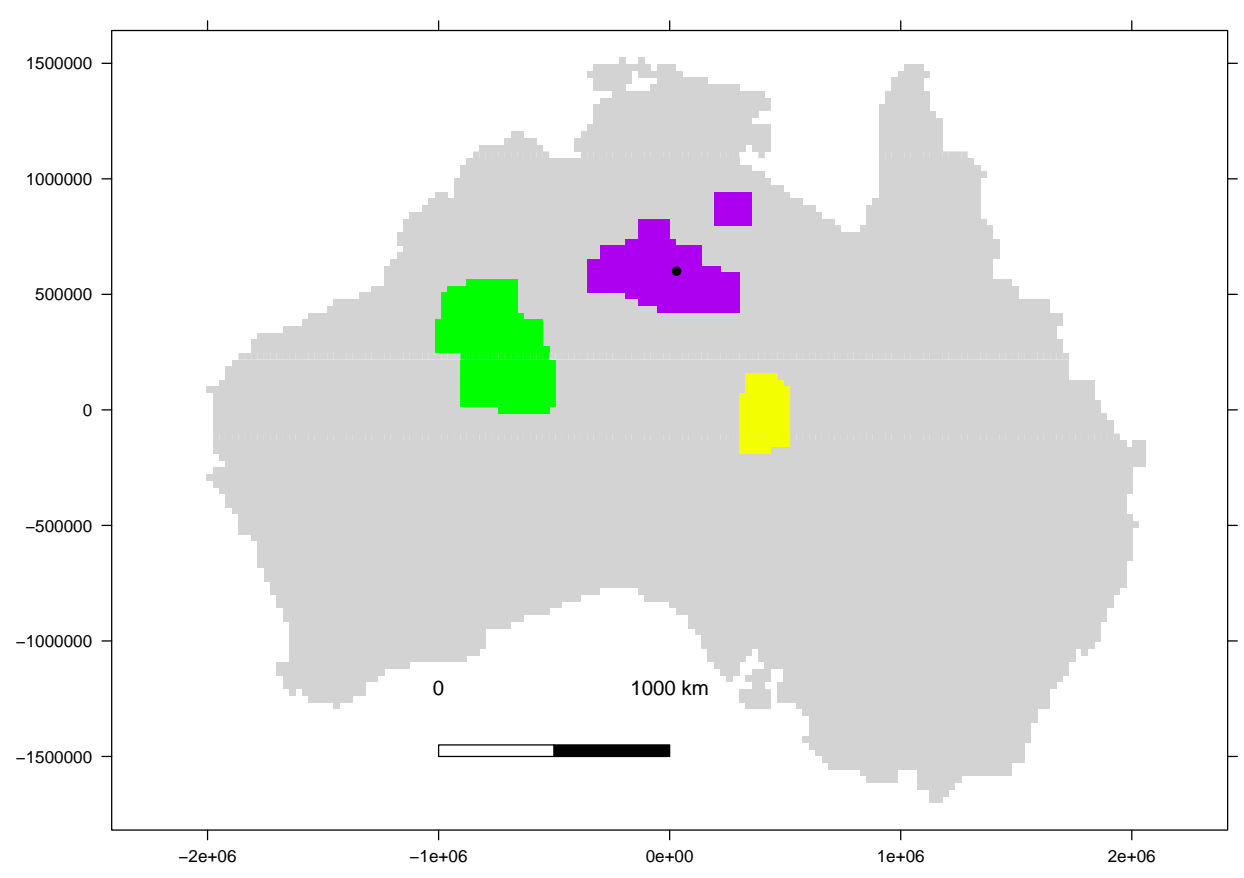

2012-11-02

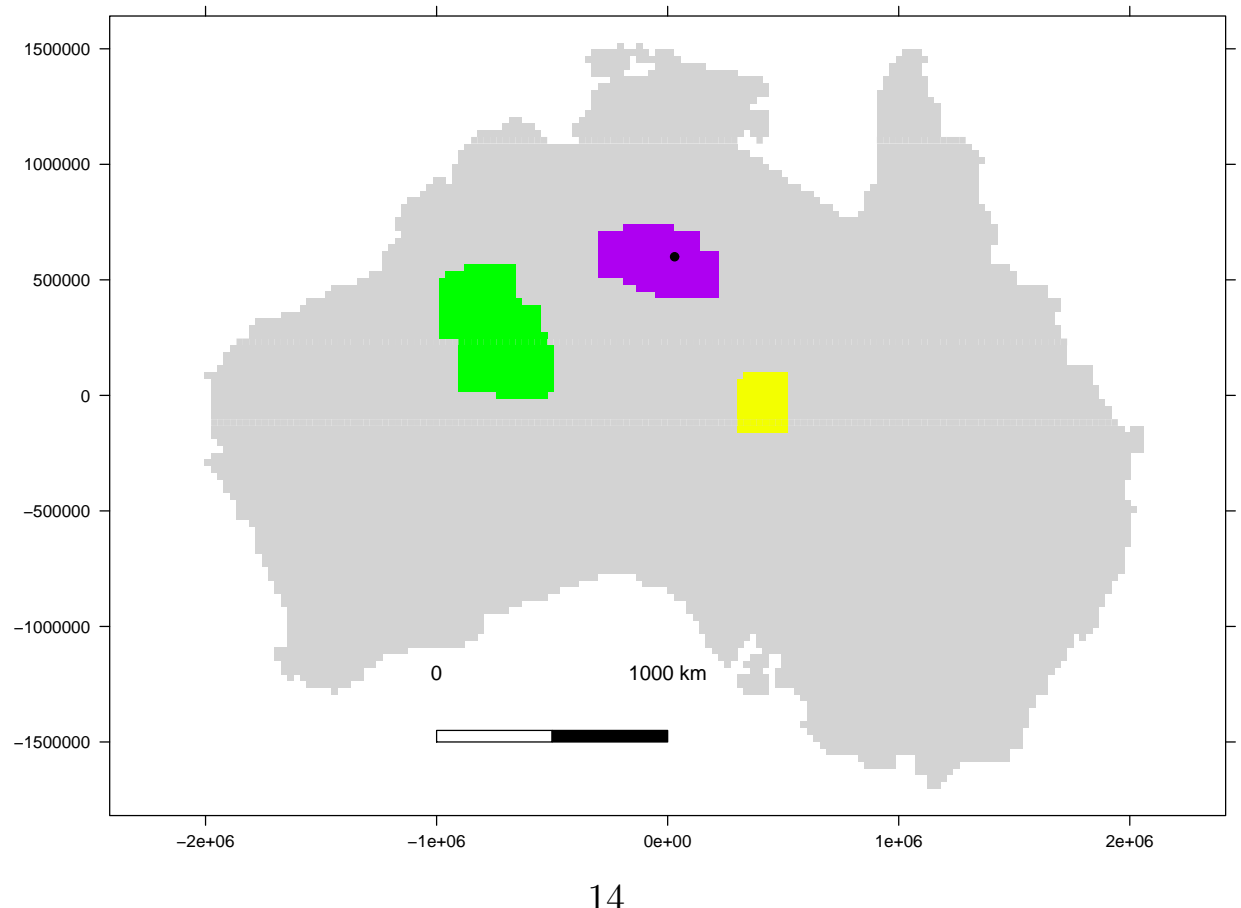

Figure 5: Drought events identified after application of an alternating sequential filter with a $3 \times 3 \times 3$ - and $5 \times 5 \times 5$-box (top panel) and a $3 \times 3 \times 3$-, a $5 \times 5 \times 5$ - and a $7 \times 7 \times 7$-box (bottom panel). From the identified drought events, only one day is shown as illustration. 
that were employed in the previous section, it was chosen to retain as many largest daily areal extents as the size $\tau$ of the structuring element in the time dimension. With respect to the characteristic reflecting the temporal component, as is already performed in many studies and is quite straightforward, the drought duration is chosen. In order to summarize the intensity reached by the drought event, the daily percentile values $q$ were first converted to survival percentiles, i.e. $1-q$, to express the intensities. In this way, higher values correspond to a higher intensity. Similarly as for the affected area, the $\tau$ largest daily intensity values were aggregated. The aggregation is performed using an ordered-weighted-averaging (OWA) operator (Yager, 1988).

The OWA operator shows a great flexibility to model a wide variety of aggregators (Ahn, 2006) as by using a different weighting vector, a different type of aggregation is performed. The OWA operator has already been used in data mining applications (Torra, 2004), decision making (Vigier et al., 2017), regression problems (Yager and Beliakov, 2010), classification (Mohammed et al., 2016) and outlier reduction (Beliakov et al., 2016). An OWA operator $F: \mathbb{R}^{n} \rightarrow \mathbb{R}$ of arity $n$ has a weighting vector $\mathbf{W}=$ $\left(w_{1}, w_{2}, \ldots, w_{n}\right)^{T} \in[0,1]^{n}$ associated with it such that $\sum_{i=1}^{n} w_{i}=1$, and takes the following form:

$$
F\left(a_{1}, a_{2}, \ldots, a_{n}\right)=\sum_{i=1}^{n} w_{i} b_{i},
$$

with $b_{j}$ the $j$-th largest element of $\left\{a_{1}, a_{2}, \ldots, a_{n}\right\}$.

If one would only be interested in the maximum (resp. the minimum) of the values, the vector of associated weights would be $\mathbf{W}=(1,0, \ldots, 0)$ (resp. $\mathbf{W}=(0,0, \ldots, 1))$. When all weights are equal, the OWA operator corresponds to the arithmetic mean. Corresponding to the weighting vector, a measure of orness of the aggregation is associated, which reflects the degree to which the aggregation behaves like an or-operator. An orness of 1, (resp. 0 ), corresponds to taking the maximum, (resp. the minimum) of the values. An orness of 0.5 corresponds to the arithmetic mean. In the current study, it was chosen to give more weight to the larger daily areal extents, therefore, an orness of 0.75 was used. The method of Fullér and Majlender (2001) was then used to obtain weights corresponding to these orness-values assuring that the dispersion of the weights is maximal, i.e. the degree to which all information in the aggregation is taken into account is maximal. Table 1 lists these weights for OWA operators of arity 3,5 and 7 .

Table 2 lists the number of completed drought events obtained after 


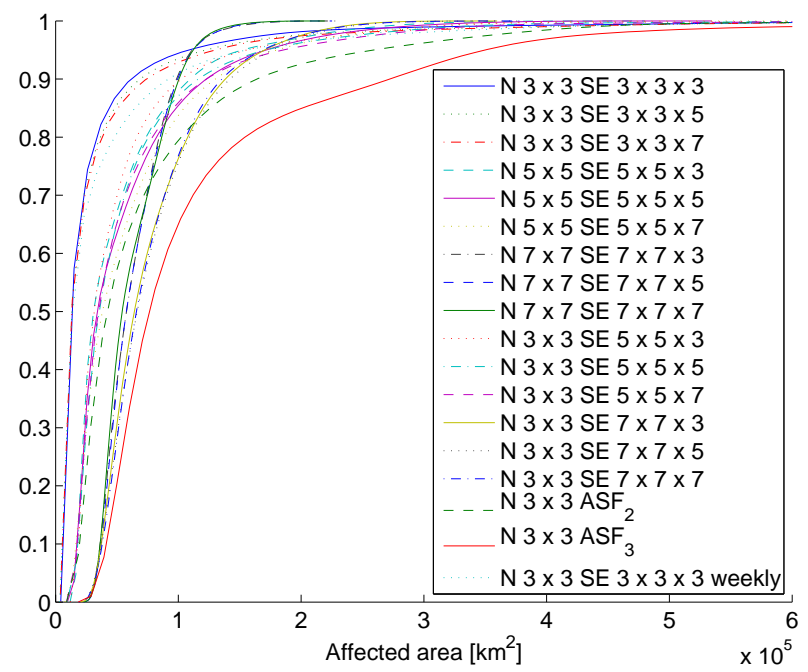

(a)

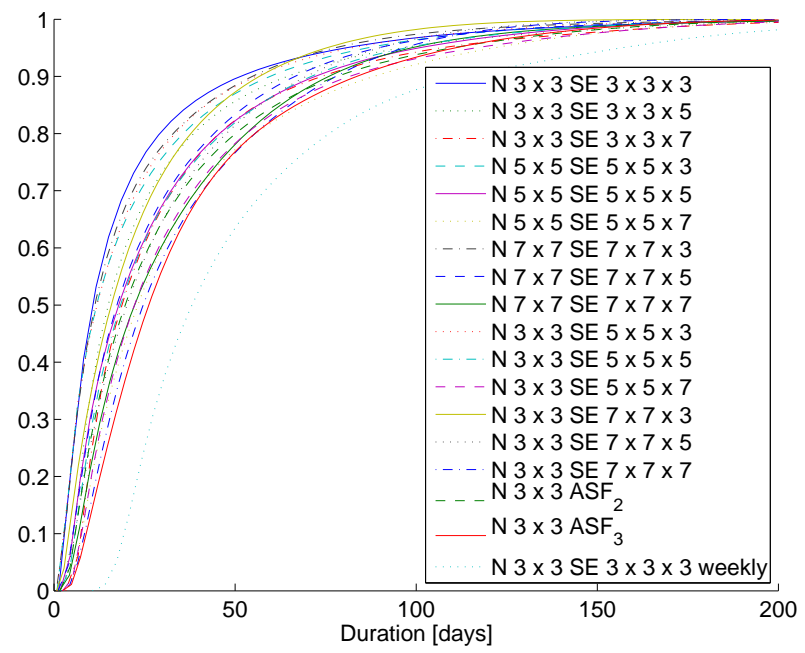

(b)

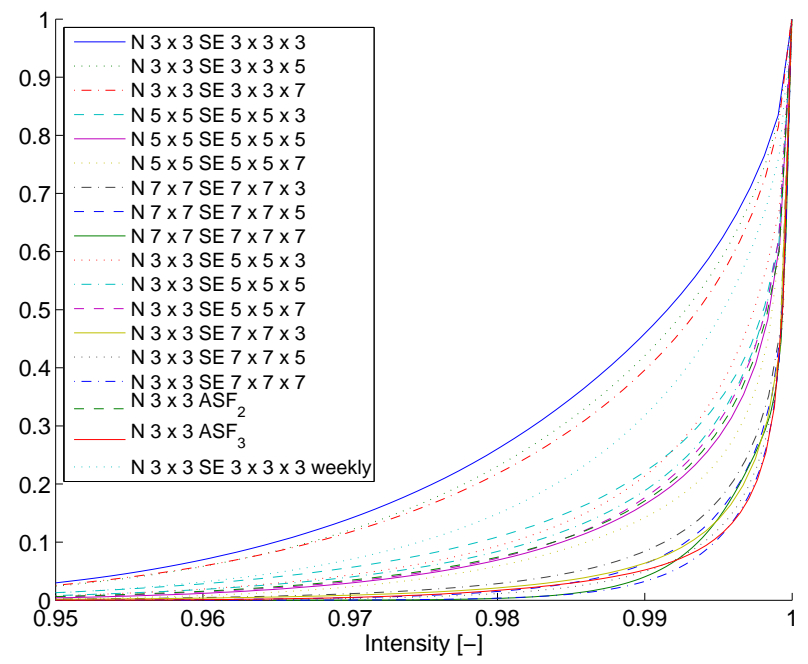

(c)

Figure 6: Cumulative distribution functions of the obtained drought characteristics for different sizes of neighbourhoods $(\mathrm{N})$, structuring elements (SE) and morphological operators. (a) Affected area, (b) Drought duration and (c) Drought intensity. 
Table 1: Weights used by the OWA operators of different arities corresponding to the length $\tau$ of the structuring element $B$ in the time dimension.

\begin{tabular}{cccc}
\hline \multicolumn{4}{c}{ orness $=0.75$} \\
\hline$\tau$ & 3 & 5 & 7 \\
\hline$w_{1}$ & 0.6162 & 0.4594 & 0.3637 \\
$w_{2}$ & 0.2676 & 0.2608 & 0.2390 \\
$w_{3}$ & 0.1162 & 0.1480 & 0.1556 \\
$w_{4}$ & - & 0.0840 & 0.1012 \\
$w_{5}$ & - & 0.0477 & 0.0659 \\
$w_{6}$ & - & - & 0.0429 \\
$w_{7}$ & - & - & 0.0279 \\
\hline
\end{tabular}

application of the morphological operators using different sizes of the structuring elements on data stemming from different neighbourhood sizes as described in the previous section. Also, the corresponding values for the first quartile, the median, the third quartile and the maximum for each of the characteristics determined on these events are listed. Figure 6 furthermore shows the cumulative distribution functions of these obtained drought characteristics. From Table 2, it can be seen that the size of the neighbourhood does not majorly influence the number of identified drought events, however, as to be expected, the larger the size of the structuring element, the lower the number of events that are identified. Furthermore, the largest drought events obtained with the smaller structuring element have a larger affected area than the largest drought events obtained with the larger structuring elements (see Table 2). Similarly, the longest drought becomes shorter when larger structuring elements are used. This is probably due to the fact that the structuring element has to fit entirely, in space and time, within the time series of thresholded maps, before a pixel being dry is retained. This also holds for the tail ends of the drought events, in which smaller structuring elements more easily fit, resulting in the fact that the largest and longest drought event is identified when a smaller structuring element is used.

Furthermore, as the use of smaller structuring elements also results in the identification of more, smaller and shorter drought events, the majority of the drought events has a smaller affected area when they are identified by smaller structuring elements. A similar observation can be made w.r.t. the duration of the events. Figure 6 shows that the drought events identified 
Table 2: Number of completed drought events for different sizes of neighbourhoods (N) and structuring elements (SE) and values corresponding to the first quartile $\left(Q_{1}\right)$, the median, the third quartile $\left(Q_{3}\right)$ and the maximum for the affected area, duration and intensity of the events. Results obtained on the basis of daily data are given in the top panel, results on the basis of weekly data are given in the bottom panel.

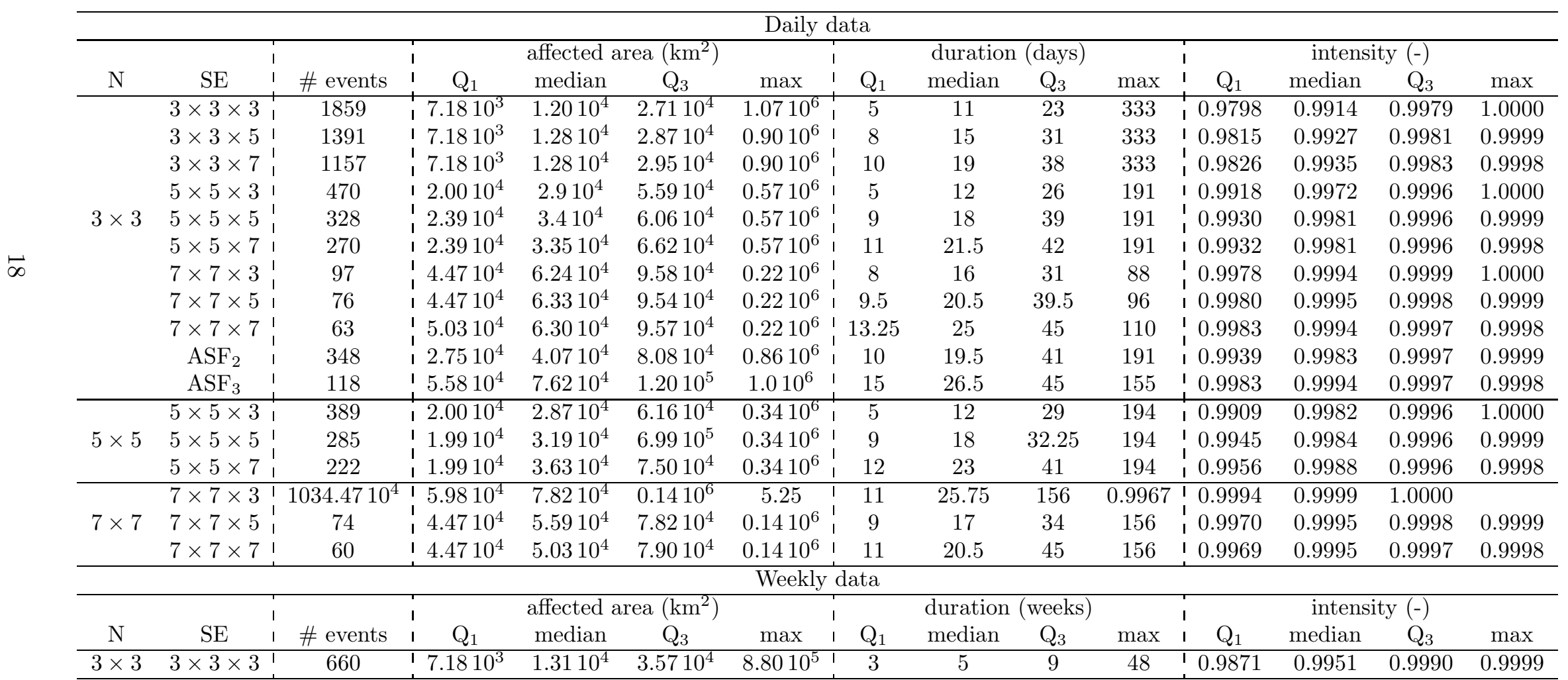


with $\mathrm{ASF}_{3}$ generally have a larger affected area. Regarding the identified drought intensities, it can be seen that the intensity values generally become larger, i.e. only the more severe drought events are retained, for larger structuring elements. It should furthermore be mentioned that the values of 1.000 in Table 2 are in fact smaller than 1, but appear as such because of the rounding. Table 2 furthermore shows that more drought events are identified after applying an ASF than after applying an open-close filter with a comparable structuring element (i.e. $\mathrm{ASF}_{2}$ vs. a $5 \times 5 \times 5$-box and $\mathrm{ASF}_{3}$ vs. a $7 \times 7 \times 7$-box). Furthermore, as expected, the affected areas become larger after application of an ASF. These observations might indicate that by using smaller structuring elements, drought events might be too easily withheld, whereas the use of larger structuring elements might be too strict. The identification of drought events by means of $\mathrm{ASF}_{2}$ or $\mathrm{ASF}_{3}$ hence might serve as a golden mean. Zhao and $\mathrm{Lu}$ (2017) also reported in their medical image analysis study that using $\mathrm{ASF}_{2}$ obtains good results for most images. Yet, these drought events are still the result of an operational procedure such that identified droughts may not entirely correspond to the opinion of water managers or on-site experience.

Generally, characterization of drought events is performed on the basis of monthly data (Byun and Wilhite, 1999). In order to compare the abovedescribed results to what could be obtained for monthly data, for each pixel its weekly average of soil moisture data was calculated. To these weekly data, a threshold of $10 \%$ was chosen for a $3 \times 3$-neighbourhood, and an open-close filter with a $3 \times 3 \times 3$-box was applied. By doing so, dry-denoted areas need a minimum duration of three weeks (more or less comparable to one month) before they will be identified as a drought event. Table 2 shows that only 660 events were identified compared to 1859 on the basis of daily data. Naturally, the majority of the events has a longer duration compared to the events identified on a daily basis. The longest drought event has a comparable duration. With respect to the affected areas and the intensities, comparable values as for the smaller structuring elements are obtained.

\section{Conclusions}

It has been shown in this paper that mathematical morphology can be used to operationally identify drought events taking into account their spatial and temporal nature. This has been illustrated using the GLEAM data set, spanning a period of 35 years of daily root-zone soil moisture values at a $25^{\circ}$ resolution. Daily data covering Australia have been selected on the basis of which soil moisture values corresponding to the 10th percentile of a 
neighbourhood have been used as threshold to determine whether drought conditions are possibly met. Operators from mathematical morphology employing a structuring element in space and time dimensions have then been applied to this spatio-temporal data series to operationally identify drought events. Different sizes of neighbourhoods and structuring elements have been used to identify drought events of which characteristics reflecting their spatio-temporal nature have been determined. Drought affected area, duration and intensity have been calculated. In order to summarize the affected area covered by and the intensity level of the drought event, an orderedweighted-averaging operator was used taking into account as many values as the time dimension of the structuring element used.

Results show that the largest and longest drought event was obtained by using the smallest structuring element. However, larger structuring elements generally identify larger, longer, and more severe drought events. Yet, less events are obtained with the larger structuring elements. This might indicate that smaller structuring elements identify drought events too easily whereas larger structuring elements are too strict in the identification of drought events. Results showed that a golden mean might be offered by applying an alternating sequential filter. However, one has to be aware that these identified drought events are the result of an operational identification procedure, from which the resulting drought events may not entirely correspond to the on-site experience or to the opinion of water managers.

In future work, it will be illustrated how the spatio-temporal characteristics of drought events as identified on the basis of mathematical morphology can be used to relate an ongoing event to historical drought events and estimate its severity by taking into account the dependence between its characteristics.

\section{Acknowledgements}

This work has been performed in the framework of the STEREO-project SR/00/302, financed by the Belgian Science Policy.

\section{References}

B.S. Ahn. On the properties of OWA operator weights functions with constant level of orness. IEEE Transactions on Fuzzy Systems, 14(4):511-515, 2006. 
K.M. Andreadis, E.A. Clark, A.W. Wood, A.F. Hamlet, and D.P. Lettenmaier. Twentieth-century drought in the conterminous United States. Journal of Hydrometeorology, 6(6):985-1001, 2005.

G. Beliakov, S. James, T. Wilkin, and T. Calvo. Robust OWA-based aggregation for data with outliers. In IEEE International Conference on Fuzzy Systems, pages 1874-1879, 2016.

H.R. Byun and D.A. Wilhite. Objective quantification of drought severity and duration. Journal of Climate, 12(9):2747-2756, 1999.

A. Dufour, O. Tankyevych, B. Naegel, H. Talbot, C. Ronse, J. Baruthio, P. Dokládal, and N. Passat. Filtering and segmentation of 3D angiographic data: Advances based on mathematical morphology. Medical Image Analysis, 17:147-164, 2013.

R. Fullér and P. Majlender. An analytic approach for obtaining maximal entropy OWA operator weights. Fuzzy Sets and Systems, 124:53-57, 2001.

D. Halwatura, A.M. Lechner, and S. Arnold. Drought severity-durationfrequency curves: a foundation for risk assessment and planning tool for ecosystem establishment in post-mining landscapes. Hydrology and Earth System Sciences, 19:1069-1091, 2015.

R.M. Haralick, S.R. Sternberg, and X. Zhuang. Image analysis using mathematical morphology. IEEE Transactions on Pattern Analysis and Machine Intelligence, 9(4):532-550, 1987.

J.E. Herrera-Estrada, Y. Sathod, and J. Sheffield. Spatiotemporal dynamics of global drought. Geophysical Research Letters, 44, 2017.

W.B. Liu, L. Wang, J. Zhou, Y.Z. Li, F.B. Sun, G.B. Fu, X.P. Li, and Y.F. Sang. A worldwide evaluation of basin-scale evapotranspiration estimates against the water balance method. Journal of Hydrology, 538:82-95, 2016.

B. Lloyd-Hughes. A spatio-temporal structure-based approach to drought characterisation. International Journal of Climatology, 32(3):406-418, 2011.

O. Lopez, R. Houborg, and M.F. McCabe. Evaluating the hydrological consistency of evaporation products using satellite-based gravity and rainfall data. Hydrology and Earth System Sciences, 21(1):323-343, 2017. 
C. Lorenz, H. Kunstmann, B. Devaraju, M.J. Tourian, N. Sneeuw, and J. Riegger. Large-scale runoff from landmasses: a global assessment of the closure of the hydrological and atmospheric water balances. Journal of Hydrometeorology, 15(6):2111-2139, 2014.

N.P. Majozi, C.M. Mannaerts, A. Ramoelo, R. Mathieu, A.E. Mudau, and W. Verhoef. An intercomparison of satellite-based daily evapotranspiration estimates under different eco-climatic regions in South Africa. Remote Sensing, 9(4), 2017.

B. Martens, D.G. Miralles, H. Lievens, R. van der Schalie, R.A.M. de Jeu, D. Fernández-Prieto, H.E. Beck, W.A. Dorigo, and N.E.C. Verhoest. GLEAM v3: satellite-based land evaporation and root-zone soil moisture. Geoscientific Model Development, 10:1903-1925, 2017.

M.F. McCabe, E. Ershadi, C. Jimenez, D.G. Miralles, D. Michel, and E.F. Wood. The GEWEX landflux project: evaluation of model evaporation using tower-based and globally gridded forcing data. Geoscientific Model Development, 9(1):283-305, 2016.

J.J. McCarthy, O.F. Canziani, N.A. Leary, D.J. Dokken, and K.S. White. Working group II: Impacts, adaptation, and vulnerability. Technical report, International Panel on Climate Change IPCC: Third Assessment Report, 2001.

T.B. McKee, N.J. Doesken, and J. Kleist. The relationship of drought frequency and duration to time scales. In Proceedings of the Eight Conference on Applied Climatology, American Meteorological Society, pages 179-184, Anaheim CA, USA, 1993.

D.G. Miralles, T.R.H. Holmes, R.A.M. De Jeu, J.H. Gash, A.G.C.A. Meesters, and A.J. Dolman. Global land-surface evaporation estimated from satellite-based observations. Hydrology and Earth System Sciences, 15:453-469, 2011.

A.K. Mishra and V.P. Singh. A review of drought concepts. Journal of Hydrology, 391:202-216, 2010.

A.K. Mishra, V.P. Singh, and V.R. Desai. Drought characterization: a probabilistic approach. Stochastic Environmental Research and Risk Assessment, 23:41-55, 2009. 
E.A. Mohammed, C.T. Naugler, and B.H. Fagler. Breast tumor classification using a new OWA operator. Expert Systems with Applications, 61:302$313,2016$.

W.C. Palmer. Meteorologic drought. Research Paper 45, US Department of Commerce, Weather Bureau, 1965.

M.J. Reddy and P. Ganguli. Application of copulas for derivation of drought severity-duration-frequency curves. Hydrological processes, 26(11):1672$1685,2011$.

T. Roy, H.V. Gupta, A. Serrat-Capdevila, and J.B. Valdes. Using satellitebased evapotranspiration estimates to improve the structure of a simple conceptual rainfall-runoff model. Hydrology and Earth System Sciences, 21(2):879-896, 2017.

F. Serinaldi, B. Bonaccosro, A. Cancelliere, and S. Grimaldi. Probabilistic characteriation of drought properties through copulas. Physics and Chemistry of the Earth, 34:596-605, 2009.

J. Serra. Introduction to mathematical morphology. Computer vision, Graphics and Image Processing, 35(3):283-305, 1986.

J. Serra and L. Vincent. An overview of morphological filtering. Circuits, Systems and Signal Processing, 11(1):47-108, 1992.

J. Sheffield and E.F. Wood. Characteristics of global and regional drought, 1950-2000: An analysis of soil moisture data from off-line simulation of the terrestrial hydrological cycle. Journal of Geophysical Research, 112: D17115, 2007.

J. Sheffield and E.F. Wood. Drought Past Problems and Future Scenarios. Earthscan, London, 2011.

J. Sheffield, G. Goteti, F. Wen, and E.F. Wood. A simulated soil moisture based drought analysis for the United States. Journal of Geophysical Research, 109:D24108, 2004.

J. Sheffield, K.M. Andreadis, E.F. Wood, and D.P. Lettenmaier. Global and continental drought in the second half of the twentieth century: Severityarea-duration analysis and temporal variability of large-scale events. Journal of Climate, 22(8):1962-1981, 2009. 
P. Soille and M. Pesaresi. Advances in mathematical morphology applied to geoscience and remote sensing. IEEE Transactions on Geoscience and Remote Sensing, 40(9):2042-2055, 2002.

V. Sridhar, K.G. Hubbard, J. You, and E.D. Hunt. Development of the soil moisture index to quantify agricultural drought and its "user friendliness" in severity-area-duration assessment. Journal of Hydrometeorology, 9(4): 660-676, 2008.

L. M. Tallaksen and H. A. J. Van Lanen. Drought as a natural hazard: Introduction. In L. M. Tallaksen and H. A. M. Van Lanen, editors, Hydrological Drought Processes and Estimation Methods for Streamflow and Groundwater, Developments in Water Science, pages 3-17. Elsevier, Amsterdam, 2004 .

K.J. Tobin and M.E. Bennett. Constraining SWAT calibration with remotely sensed evapotranspiration data. Journal of the American Water Resources Association, 53(3):593-604, 2017.

V. Torra. OWA operators in data modeling and reidentification. IEEE Transactions on Fuzzy Systems, 12(5):652-660, 2004.

P. Trambauer, E. Dutra, S. Maskey, M. Werner, F. Pappenberger, L.P.H. van Beek, and S. Uhlenbrook. Comparison of different evaporation estimates over the African continent. Hydrology and Earth System Sciences, 18(1): 193-212, 2014.

H.P. Vigier, V. Scherger, and A. Terceno. An application of OWA operators in fuzzy business diagnosis. Applied Soft Computing, 54:440-448, 2017.

G. Wong, M.G. Lambert, M. Leonard, and A.V. Metcalfe. Drought analysis using trivariate copulas conditional on climatic states. Journal of Hydrologic Engineering, 15(2):129-141, 2010.

R.R. Yager. Ordered weighted averaging aggregation operators in multicriteria decision making. IEEE Transactions on Systems Man and Cybernetics, 18:183-190, 1988.

R.R. Yager and G. Beliakov. OWA operators in regression problems. IEEE Transactions on Fuzzy Systems, 18(1):106-113, 2010.

W. Zhao and H. Lu. Medical image fusion and denoising with alternating sequential filter and adaptive fractional order total variation. IEEE Transactions on Instrumentation and Measurement, 9(66):2283-2294, 2017. 
${ }_{580}$ J. Zscheischler, M.D. Mahecha, S. Harmeling, and M. Reichstein. Detection 581 and attribution of large spatiotemporal extreme events in earth observa582 tion data. Ecological Informatics, 15:66-73, 2013. 